\section{Silver Scalpel Award Winner}

\author{
Supplied by the British Association of Oral and Maxillofacial Surgeons (BAOMS)
}

\section{Manjit Dhillon, a Consultant in Oral \& Maxillofacial Surgery (OMFS) at Aberdeen Royal Infirmary, has won this year's Association of Surgeons in Training (ASiT) Silver Scalpel Award. In Scotland, she is the first Asian female Consultant in the specialty and the first woman to sub-specialise in head and neck oncology and reconstructive microvascular surgery.}

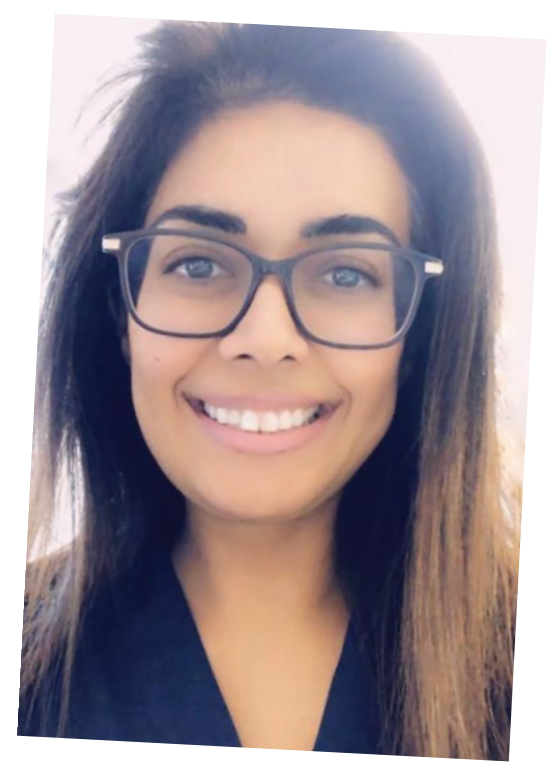

obtained her Bachelors of Dental Surgery with Honours at the University of Dundee. She went on to qualify in Medicine with Commendation at the University of Aberdeen, and was awarded the Dyce Davidson Medal in Clinical Medicine as the top student in her finals exam. Her specialty training experience in OMFS was obtained between the Oxford and Scotland training programmes. She was awarded Fellow of the Royal College of Surgeons \& Physicians in Glasgow in 2013.

The British Association of Oral and Maxillofacial Surgeons (BAOMS) spoke to Manjit about her career and background.

\section{What attracted you to surgery in the first place?}

I am instinctively a caring and empathic person, so I was always drawn towards the caring professions. That, coupled with my fascination of the inner workings of the human body, made surgery an obvious choice. I love performing surgery. Watching the layers of anatomy unfold is one of the most beautiful living art forms. It never ceases to amaze me how remarkable and ingenious the human machine is.

\section{What is the best part of being a surgeon?}

As a clinician, it is a huge privilege for patients to put their total trust in you for their lives. It is fulfilling and hugely satisfying to be able to help others, relieve suffering and making a positive difference to the lives of patients and their families.

As a trainer, it is an indescribable joy and sense of achievement to be part of the growth and professional development of trainees, their successes and their journey in becoming proficient in their craft.

How does it feel to have won the prestigious ASiT 2021 Silver Scalpel award and what does it mean to you to receive it? I was thrilled and surprised to have been nominated, but to have won is such an honour. For me, the award is a reflection of the inspirational and amazing team that I work with. They are the ones that go the extra mile every day for patients, each other and me. It is because of my team that I get to be part of many extraordinary experiences and achievements. They have been like a family to me and I'm very fortunate to have their support.
The award is also a tribute to my own trainers and my family who supported, encouraged and believed in me. Without them, I wouldn't be here.

\section{Do you think winning the award will inspire other women to become OMFS surgeons?}

I hope I inspire and empower anyone who has an ambition to be a surgeon, no matter what their background, race, colour, gender, sexual orientation, social status, religion or ability.

There are currently three women out of 38 OMFS consultants in Scotland. There are not only unique challenges and barriers for women in surgery, but also for many other individuals with diverse characteristics. The profession is much more aware of these challenges now, more than ever, with a much greater commitment towards encouraging diversity and inclusivity in healthcare.

\section{How did your surgical training shape you?}

As a very ordinary person, from a humble background, my surgical training experience did not only teach me my craft, but it was also formative in making me the person I am today. Surgical training gave me a deep appreciation and understanding of human behaviours as well as an insight into the vulnerabilities and fragilities that can affect care providers and trainees, as well as patients.

Healthcare workers are heroes of the NHS, who constantly put their patients' wellbeing before their own. But we often forget that they are not exempt from the human condition. I learned that supporting and looking after the team and 'caring for the carers' is vital to providing excellence in patient care.

\section{What are the challenges of a career in surgery?} A surgical career is challenging for everyone in many different ways. There are the obvious, stressors of the long hours, the heavy responsibility of performing high-risk complex surgery, the constant displacement and unsettlement whilst moving around on placements, the financial burden of funding a second degree, 
1 and we must not forget the sacrifices that families make for our careers. These are all stressful things to go through for everyone.

But for some, there are added pressures and hurdles that are less conspicuous and make the training experience more challenging, demanding and sometimes distressing. Some face personal life hardships, physical and mental health issues, workplace bullying, burnout, discrimination and other unacceptable workplace inequalities. All healthcare workers, in particular leaders and trainers in the profession, need to be aware of these visible and invisible obstacles and support trainees and colleagues fairly, without judgement.

\section{What did trainees, colleagues and the Association of Surgeons} in Training (ASiT) see in your practice that went beyond the usual professional standards?

My interest in human factors, along with my own experience, has given me insight into how systemic weaknesses, non-technical failures, toxic cultures, poor behaviours and biases can have a detrimental impact on staff/trainee performance and experience. As a result, my focus has not only been on patient care but also on the wellbeing of the staff and team. I try to promote an open, just, fair and inclusive culture for my teams, where the hierarchy gradient is flattened and everyone is equal, feels valued and their voice heard.

In recognition of my own weaknesses and failings, I encourage a growth mindset, where the whole team learns from errors and failures without fear of blame, reprisal or being undermined.

\section{'I try to promote an open, just, fair and inclusive culture for my teams, where everyone is equal'}

\section{How do you provide support to your trainees?}

Colleagues and trainees value having someone they can trust, in a team where they feel accepted and not judged. I want trainees to see me as a part of their support network where I am not only their trainer and mentor but also a friend who genuinely cares.

As the caring profession, we need to show 'beneficence, non-maleficence and primum non nocere' to our colleagues and trainees, as well as our patients.

We should strive to be the best we can be as clinicians but of equal import is to strive to be the best we can be as caring human beings.

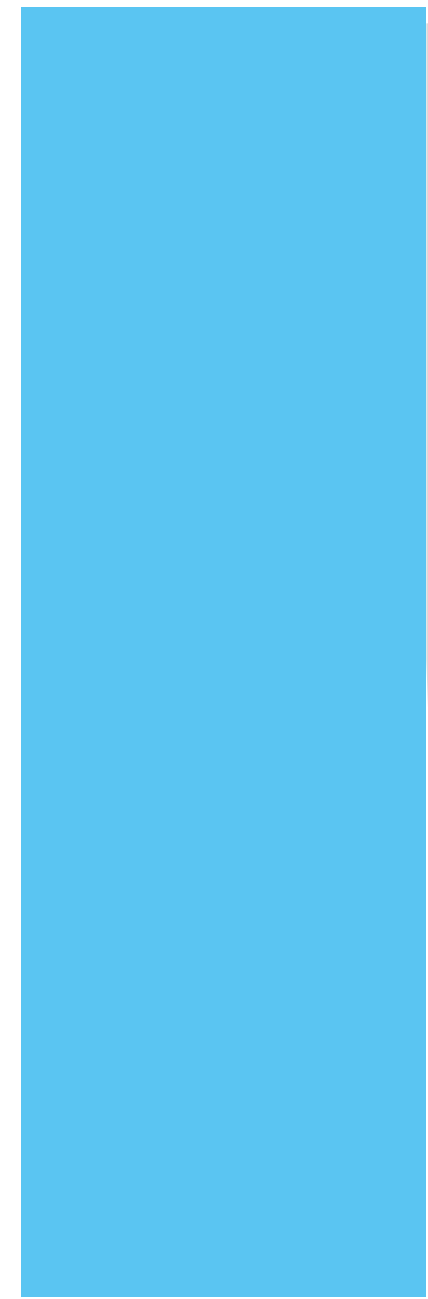

